\title{
Image Guided Surgery System for Abdominal Aortic Aneurysm Operation
}

\author{
Ming $\mathrm{Niu}^{1, \mathrm{a}}$, Yajun $\mathrm{Wu}^{1}$, Lisheng $\mathrm{Wang}^{1}$, Chaoyi Cui ${ }^{2, \mathrm{~b}}$ \\ ${ }^{1}$ Institute of Image Processing and Pattern Recognition, Department of Automation, Shanghai Jiao \\ Tong University, Shanghai, China
}

${ }^{2}$ The Ninth People's Hospital affiliated to Shanghai Jiao Tong University, Shanghai, China

amingknight@sjtu.edu.cn, bcuichoi8432@163.com

Keywords: Abdominal aortic aneurysm, segment, reconstruct, surgery planning.

\begin{abstract}
Abdominal aortic aneurysm (AAA) is a local enlargement of the abdominal aortic. It can bring $90 \%$ mortality rate when the aneurysm expands gradually until rupture. Clinically, the stent interventional procedure is an effective means of treating the disease, which requires clinicians clearly understand the anatomical structure of abdominal aorta and the aneurysm. In order to reduce the measurement risk and enhance the safety of operation, an image guided surgery (IGS) system is applied into operation. By the system, surgeons can know exactly morphological structure about the lesions and extract concerned parameters during surgery. This paper introduces how 3D models of the abdominal aorta and aneurysm are segmented and reconstructed from CT images, and how appropriate centerline path are extracted and used for internal virtual roaming in these 3D models. Then how tumor diameter is measured through cutting plane perpendicular to computed axis Clinical applications have shown that such IGS system is practical and helpful in the operation.
\end{abstract}

\section{Introduction}

With high blood pressure, arteriosclerosis and other high risk factors due to the fact of aging population, numbers of cases with abdominal aortic aneurysm (AAA) is increasing rapidly in recent decades. As a common and severe vascular disease, AAA is typically local lesion expansion and lesion diameter of the aorta is significantly than normal [1]. Clinical researches demonstrate that the stent interventional procedure is an effective treatment for AAA [2]. The surgery involves key steps including estimating the anatomical structure and size of the aneurysm and measuring abdominal aortic bifurcation angle. A feasible solution to such problems is to apply an appropriate image guided surgery (IGS) system into the operation.

In a typical IGS system, detailed patient specific anatomic models are usually segmented and reconstructed properly. The actual patient tissues and organs can be registered with the reconstructed models by moving and tracking surgical instruments [3]. The surgeons can get real-time feedback about the relationship between the relative position of the instrument and surrounding anatomic structures [4]. In the case of abdominal aortic aneurysm stent interventional procedures (SIP), IGS can be used to locate the aneurysm in real-time. And in IGS system for SIP, surgeons can also observe internal structure with the assistance of virtual camera during the virtual roaming procedure.

In this paper, a semi-auto segmentation method with some interactive operation is put into use to segment target structure from CT images. Then 3D models are reconstructed using improved surface rendering method. Subsequently, the centerline is extracted by first computing the Voronoi diagram of the aorta surface model as approximation of the medial axis and then computing the minimum cost path between starting point and ending point on the medial axis using Fast Marching algorithm [5] [6]. By determining parameters of the virtual camera, the internal virtual roaming of aorta and the aneurysm can be achieved. For the purpose of tumor diameter measurement, virtual cutting plane at any angle can be obtained as interactive feature in the IGS system. Actual clinical applications have demonstrated that such IGS system is practical and useful in the operation. 


\section{Methods}

Firstly, this paper introduces a level-set image segmentation algorithm and put it into use of the abdominal aorta and aneurysm segmentation from CT images [7]. And surface model will be reconstructed using surface rendering method based on segmentation results. Secondly, we extract a centerline as the advance path and determine some virtual camera parameters for internal virtual roaming. Thirdly, we obtain certain virtual cutting planes at any angle and display them in screen, which can act as supplied displaying view instead of traditional view along the axial, sagittal and coronal planes. And we can also at any angle with assistance of virtual planes. The overall process is as follows:

Segmentation and reconstruction of the abdominal aorta and aneurysm. In our case of abdominal aorta and aneurysm segmentation, the morphology variety of aorta among patients, the disturbing bifurcations and anatomical structures around the aorta both bring much difficulty to the segmentation process. In our IGS system, the Fast Marching level set method is selected to segment the abdominal aorta and the aneurysm, which can deal with the interference of gray scale and noise as well as reserve topological details during the evolution of surfaces.

Boundary curve on a two-dimensional plane or boundary surface on three-dimensional space both can be regard as zero level set of a higher dimensional space level set function, while the evolution of level set function within the constraints of differential equations .Therefore, evolution of the border can be obtained by solving the zero level set. The level set is always defined with implicit function. The zero level set move along the gradient direction which is exactly Hamilton-Jacobi equation and evolution of object boundaries can be obtained by solving this partial differential equation. Another problem that should be pay attention to is updating zero level set in global domain require large amount of computation. One feasible solution is only taking the pixels within a narrow range of current border by fast marching method. The growth order of calculation can be reduced from $O\left(N^{3}\right)$ to $O(N \log N)$.

Generally, there are three key points in fast marching level set segmentation method including initial value of the level set function, form of speed function and updating zero level set. In our method, it can be expressed as following four basic steps.

Step1.apply Gaussian low-pass filter to the original image for removing image noise.

Step2.compute gradient and put it into use of sigmoid function, which can widen interest gray scale range through nonlinear transformation. Then we get target Speed Image.

Step3.select seed points manually in the three-dimensional tomographic slice as initial position of the evolution boundaries, and set stopping time to generate Time Crossing Map (optimal value is 50 in our system).

Step4.select proper crossing time based on Time Crossing Image to determine segmentation area, and mask on the original image to get the final segmentation results.

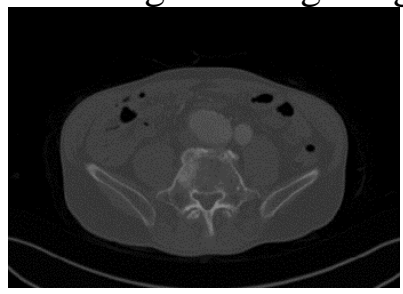

(a)

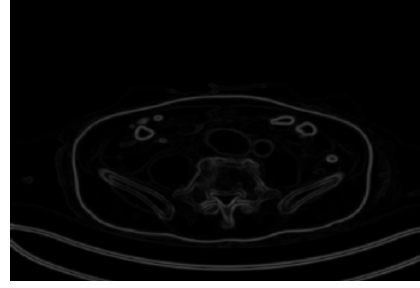

(b)

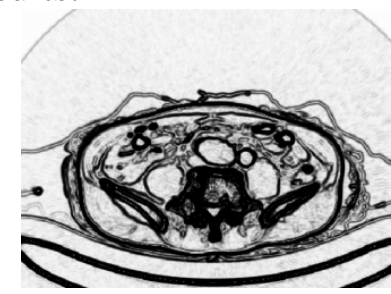

(c)

Fig 1. (a) Original DICOM slice and (b) its gradient image after Gaussian smoothing filter with $\sigma=1.2$. (c) The sigmoid filtered image of $(b)($ alpha $=-10.0)$. 


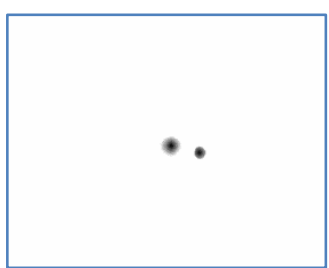

(a) $T_{S}=10$

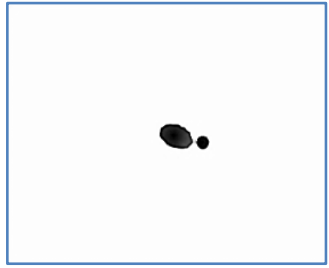

(d) $T_{S}=50$

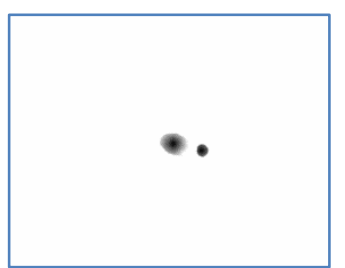

(b) $T_{s}=20$

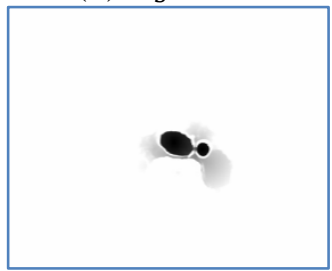

(e) $T_{s}=100$

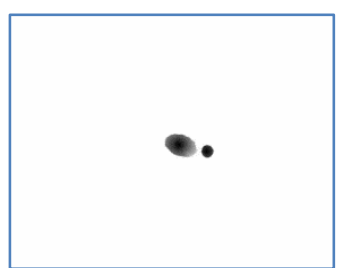

(c) $T_{s}=30$

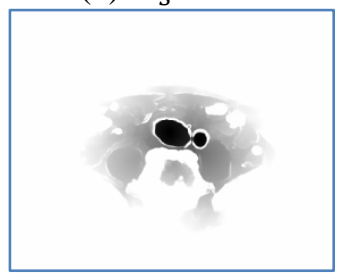

(f) $T_{S}=200$

Fig 2. Time-Crossing Map with different stopping time (the value increase from 10 to 200).

Fig 1 (a)...(c) reveals the generation process of speed image. And it should be noticed that the alpha parameter of sigmoid function is better to be set as negative, which can achieve so-called anti-color effect and is beneficial for subsequent processing.

Fig 2 illustrates the effect of different stopping time. By six contrast time-crossing map we can find that the target area for segmentation has been nearly complete when $T_{s}$ is 50 . When $T_{S}$ increase to 100 , the target area boundary is still not arrived indicates that speed image generated from gradient feature can inhibit the evolution boundary. When $T_{s}$ is set to 200, the evolution boundary have arrived some non-target area while some portions of the target area boundary still has not been get.

After getting segmentation results, surface rendering method is chosen for reconstruction step instead of volume rendering method. In order to retain more local details, volume rendering method needs traversal all voxels and cause huge computational overhead. Considering actual rendering speed of our IGS system, surface rendering will be more proper for final reconstruction model.

Centerline extraction and internal virtual roaming based on reconstructed $3 \mathrm{D}$ model. The abdominal aorta and aneurysm is tube-like structure on the whole. Therefore a centerline of the 3D model can be selected as the virtual roaming path. Various centerline extraction methods have been proposed in past decades. Basically, these methods can be divided into two classes, one is based on three-dimensional volume data, and the other is based on three-dimensional boundary surface. In our IGS system, the latter method is applied into centerline extraction. And the specific implementation is finding least-cost path in Voronoi diagram [9].

Firstly, we can calculate the medial axis for the closed tubular tissue represented by boundary surfaces using computer graphics methods. According to definition of Voronoi diagram, the distances of any point on it to all recent sampling points are equal. Therefore the Voronoi diagram can be regarded as an equivalent axial. According to duality between the Voronoi diagram and Delaunay Triangulation, we can get the Voronoi region boundary set of surface rendering 3D model with the closed triangular surface models for Delaunay Triangulation. After the above steps, an approximate medial axis can be obtained and the corresponding distance field boundary can also be constructed at the same time, which consists of all inscribed sphere radius of each point in medial axis. 


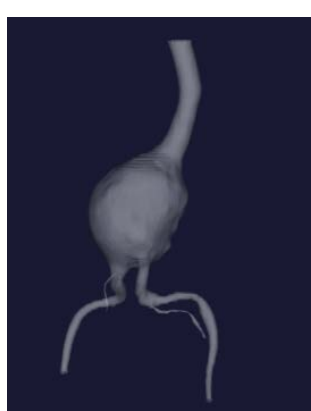

(a)

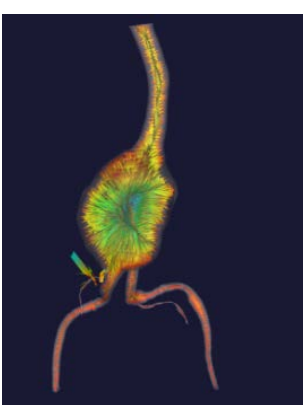

(b)

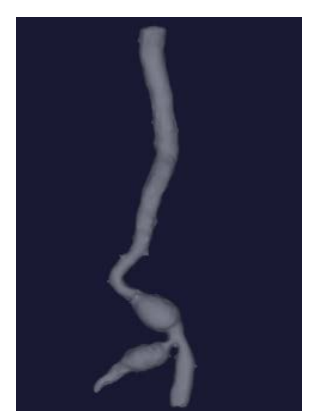

(c)

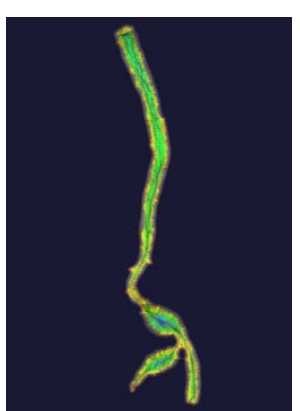

(d)

Fig 3. The surface rendering model and its Voronoi diagram of abdominal. The color from shallow to deep represents corresponding boundary distance from small to big in color map.

Secondly, set starting and ending points manually considering interference of local vascular bi-furcation. And calculate arrival time from the start point of arriving at any point in medial axis. Then starting from the end point and backtracking along the gradient direction of arrival time, we can get corresponding centerline from the track.

After completing the centerline extraction, we carry out equidistant sampling on the centerline and apply the down-sampling point as a roaming connection path. Then determine certain camera parameters such as camera focus, camera view and the camera towards clipping plane and begin final virtual roaming.

Interactively cutting virtual plane at any angle and measuring concerned parameters. In order to measure the aneurysm with irregular shape, the system allows select the virtual tomographic slices at any angle interactively. A virtual slice is in fact a plane, which can be uniquely determined by a point and the normal vector. In actual operation, a fixed step size that indicates the smallest movement in corresponding direction will be set. Gray value anywhere on actual virtual slice is obtained by mapping 2D coordinates to 3D space and calculating original volume data with linear interpolation.

\section{Results}

We evaluate our method and the IGS system by some real clinical medical image data from patients. The abdominal aorta and aneurysm can be segmented and reconstructed by the method integrated into the IGS system. And by extracting centerline of reconstructed 3D model as path, complete virtual roaming process can be achieved. The clinical surgeons also measure the aneurysm by selecting virtual slice and get relatively accurate result. The clinical surgeons think that the accuracy of measuring is acceptable, and such IGS system is practical and helpful in guiding the surgery. Next we put some intermediate processing results.

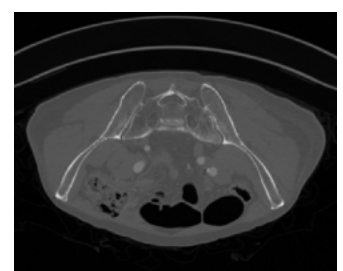

(a)

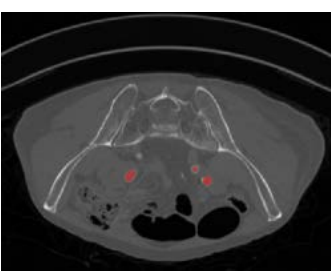

(b)

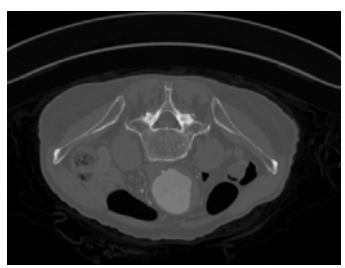

(c)

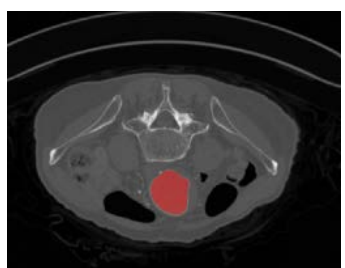

(d)

Fig 4. Original images and their corresponding segmentation results, among them (a) and (b) (No.23), (c) and (d)

(No.65). 


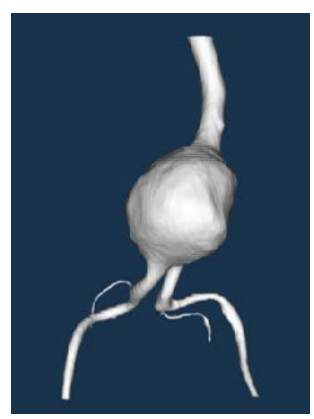

(a)

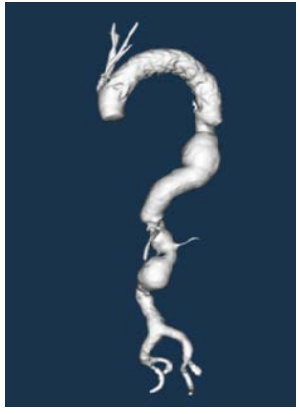

(b)

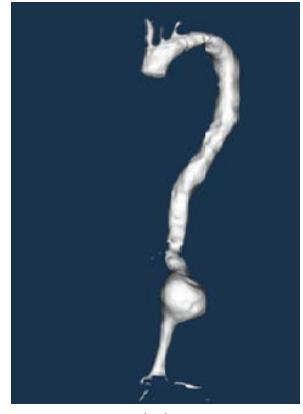

(c)

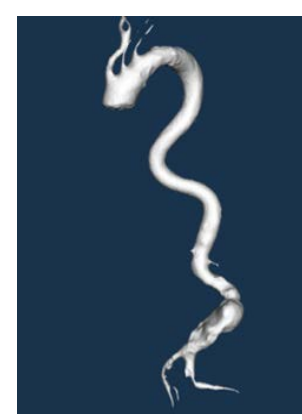

(d)

Fig 5. Surface rendering models the abdominal aorta and aneurysm

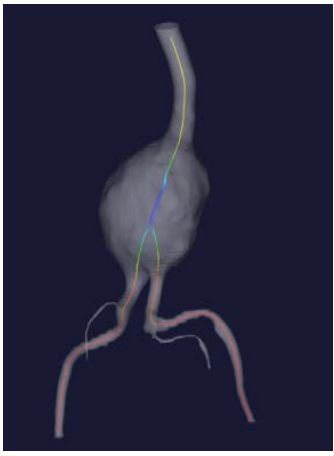

(a)

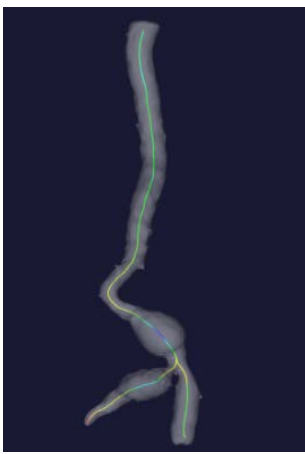

(b)

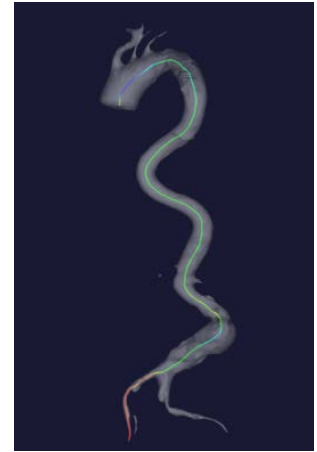

(c)

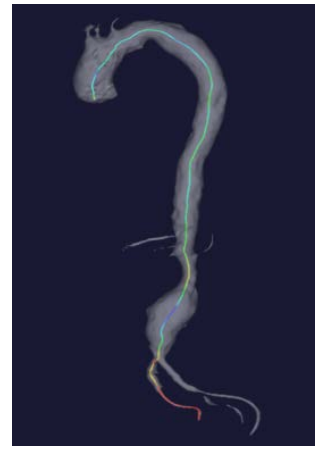

(d)

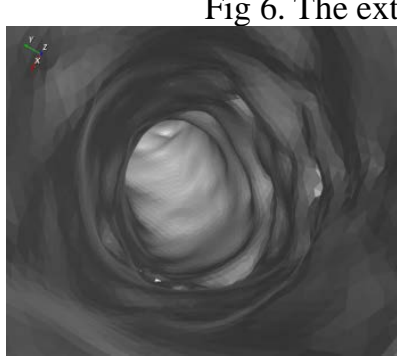

(a)

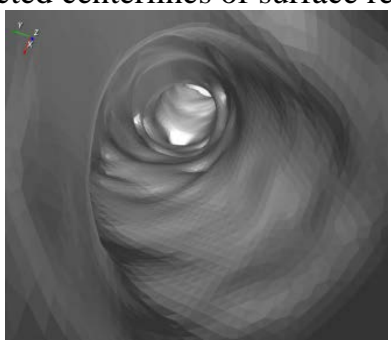

(b)

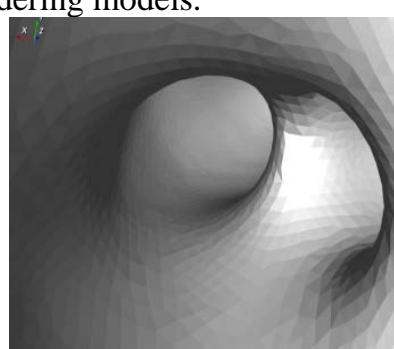

(c)

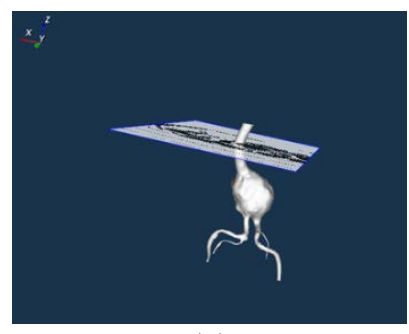

(a)

Fig 7. Virtual roaming scene in abdominal aorta.

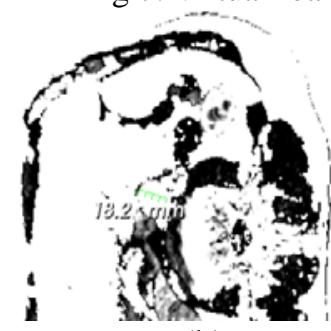

(b)

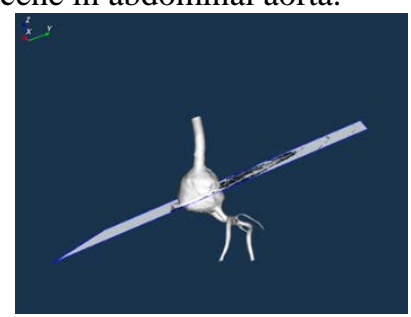

(c)

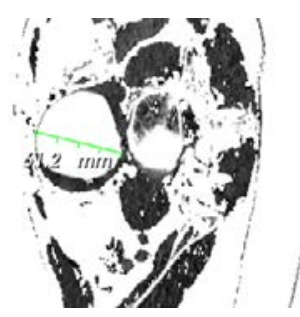

(d)

Fig 8. Interaction of the virtual slice on 3D reconstructed model and tumor diameter measurement on mapped 2D plane.

\section{Conclusion}

This paper introduces an IGS system are built and applied into the operation for AAA. In the system, 3D models of the abdominal aorta and aneurysm are segmented and reconstructed from raw CT images. And with extracted centerline, virtual roaming process can be carried out in reconstructed 3D model. The system also provides surgeons accessibility for selecting virtual slice interactively. And tumor diameter can be measured on virtual plane. Clinical experiments have shown the validity and feasibility of our techniques in our IGS system for the operation.

\section{Acknowledgement}

This work was supported in part by NSFC of China (61375020), 973 program of China 
(2013CB329401) and Cross Research Fund of BME of SJTU (YG2013ZD02).

\section{References}

[1] Sakalihasan, N., Limet, R., \& Defawe, O. D. (2005). Abdominal aortic aneurysm. The Lancet, 365(9470), 1577-1589.

[2] Prinssen, M., Verhoeven, E. L., Buth, J., Cuypers, P. W., van Sambeek, M. R., Balm, R., ... \& Blankensteijn, J. D. (2004). A randomized trial comparing conventional and endovascular repair of abdominal aortic aneurysms. New England Journal of Medicine, 351(16), 1607-1618.

[3] Blum, U., Voshage, G., Lammer, J., Beyersdorf, F., Töllner, D., Kretschmer, G., ... \& Thurnher, S. (1997). Endoluminal stent-grafts for infrarenal abdominal aortic aneurysms. New England Journal of Medicine, 336(1), 13-20.

[4] Rubin, G. D., Beaulieu, C. F., Argiro, V., Ringl, H., Norbash, A. M., Feller, J. F., ... \& Napel, S. (1996). Perspective volume rendering of CT and MR images: applications for endoscopic imaging. Radiology, 199(2), 321-330.

[5] Kühnapfel, U., Cakmak, H. K., \& Maßß, H. (2000). Endoscopic surgery training using virtual reality and deformable tissue simulation. Computers \& graphics, 24(5), 671-682.

[6] Li, C., Xu, C., Gui, C., \& Fox, M. D. (2010). Distance regularized level set evolution and its application to image segmentation. Image Processing, IEEE Transactions on, 19(12), 3243-3254.

[7] Adalsteinsson, D., \& Sethian, J. A. (1999). The fast construction of extension velocities in level set methods. Journal of Computational Physics, 148(1), 2-22.

[8] Wang, L. M., Zhao, Y. L., Chen, F.L., \& Han, Y. (2011). The 3D CT reconstruction algorithm to directly reconstruct multi-characteristic based on EMD. Measurement, 44(10), 2043-2048.

[9] Bradshaw, G., \& O’Sullivan, C. (2004). Adaptive medial-axis approximation for sphere-tree construction. ACM Transaction on Graphics (TOG), 23(1), 1-26.

[10]Attali, D., Boissonna, J. D., \& Edelsbrunner, H. (2009). Stability and computation of medial axes-a state-of-the-art report, Mathematical foundations of scientific visualization, computer graphics, and massive data exploration, 6, 109-125. 